BMC

Genomics

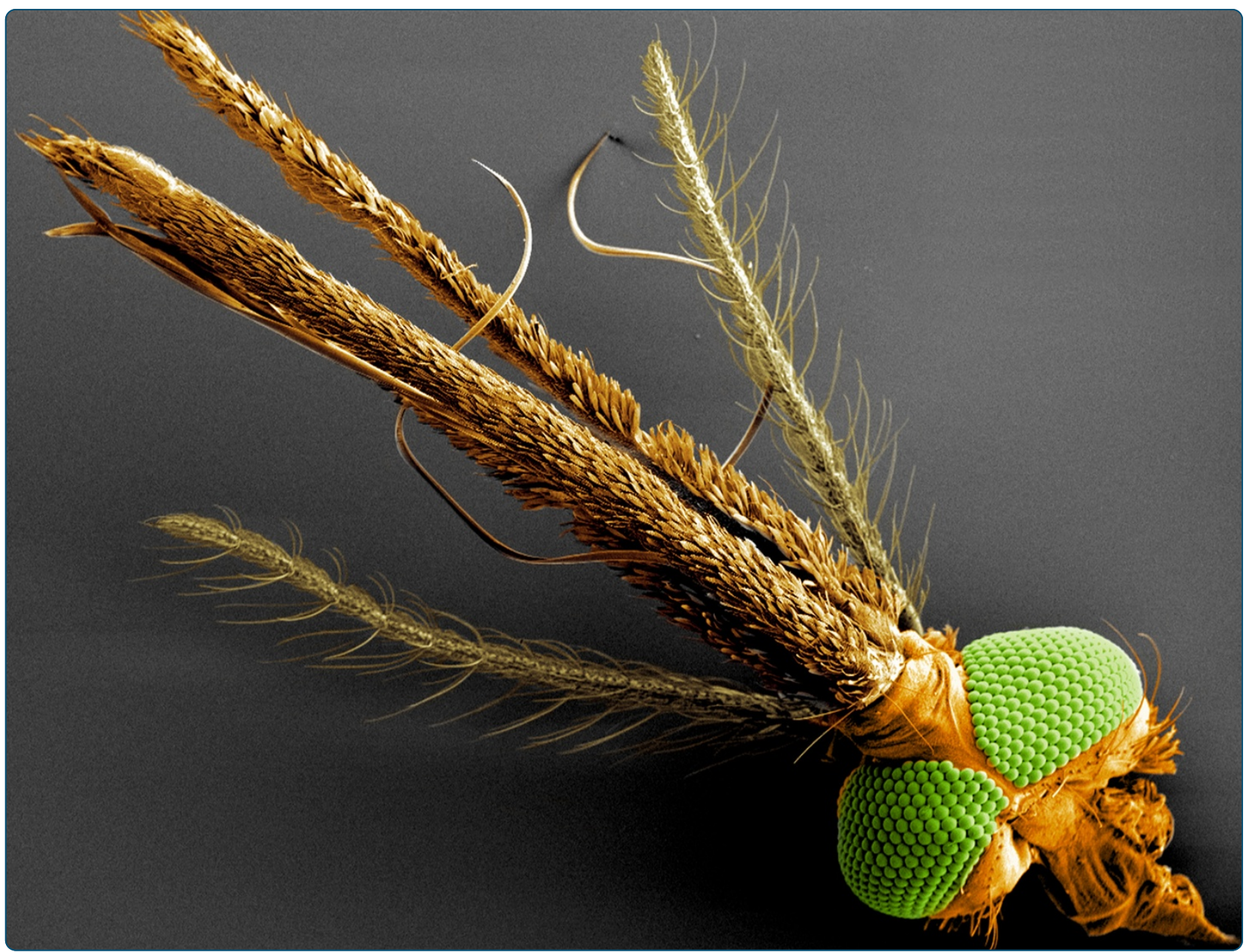

Antennal transcriptome profiles of anopheline mosquitoes reveal human host olfactory specialization in Anopheles gambiae

Rinker et al. 


\title{
Antennal transcriptome profiles of anopheline mosquitoes reveal human host olfactory specialization in Anopheles gambiae
}

David C Rinker ${ }^{1+}$, Xiaofan Zhou ${ }^{2+}$, Ronald Jason Pitts ${ }^{2}$, The AGC Consortium, Antonis Rokas ${ }^{1,2}$ and Laurence J Zwiebel ${ }^{1,2,3^{*}}$

\begin{abstract}
Background: Two sibling members of the Anopheles gambiae species complex display notable differences in female blood meal preferences. An. gambiae s.s. has a well-documented preference for feeding upon human hosts, whereas An. quadriannulatus feeds on vertebrate/mammalian hosts, with only opportunistic feeding upon humans. Because mosquito host-seeking behaviors are largely driven by the sensory modality of olfaction, we hypothesized that hallmarks of these divergent host seeking phenotypes will be in evidence within the transcriptome profiles of the antennae, the mosquito's principal chemosensory appendage.

Results: To test this hypothesis, we have sequenced antennal mRNA of non-bloodfed females from each species and observed a number of distinct quantitative and qualitative differences in their chemosensory gene repertoires. In both species, these gene families show higher rates of sequence polymorphisms than the overall rates in their respective transcriptomes, with potentially important divergences between the two species. Moreover, quantitative differences in odorant receptor transcript abundances have been used to model potential distinctions in volatile odor receptivity between the two sibling species of anophelines.

Conclusion: This analysis suggests that the anthropophagic behavior of An. gambiae s.s. reflects the differential distribution of olfactory receptors in the antenna, likely resulting from a co-option and refinement of molecular components common to both species. This study improves our understanding of the molecular evolution of chemoreceptors in closely related anophelines and suggests possible mechanisms that underlie the behavioral distinctions in host seeking that, in part, account for the differential vectorial capacity of these mosquitoes.
\end{abstract}

Keywords: Anopheles, Mosquito, Antenna, Transcriptome, Olfaction, Malaria, Host-seeking, Odorant receptor, Molecular evolution, RNAseq

\section{Background}

Anopheles gambiae sensu stricto is the major sub-Saharan vector for the human malaria parasite Plasmodium falciparum and the nominotypical member of a set of morphologically indistinguishable species that comprise the Anopheles gambiae complex [1]. The two molecular forms of An. gambiae s.s. (M and S), along with Anopheles

\footnotetext{
*Correspondence: I.zwiebel@vanderbilt.edu

${ }^{\dagger}$ Equal contributors

${ }^{1}$ Center for Human Genetics Research, Vanderbilt University Medical Center,

Nashville, Tennessee, USA

${ }^{2}$ Department of Biological Sciences, Vanderbilt University, Nashville,

Tennessee, USA

Full list of author information is available at the end of the article
}

arabiensis, constitute the major malaria vectors within this species complex. Despite their close evolutionary relationship, other members of the complex display either little (Anopheles merus, Anopheles melas and Anopheles bwambae) or no (Anopheles quadriannulatus A and Anopheles quadriannulatus B) vectorial capacity for human malaria [2].

Interestingly, the sole non-vector member of this species complex, An. quadriannulatus nevertheless is competent for P. falciparum infection [3,4] and molecular evidence suggests that the karyotype for this species derived directly from that of the main vector An. gambiae s.s. [5]. However, An. quadriannulatus is still considered to

\section{Biomed Central}

(c) 2013 Rinker et al.; licensee BioMed Central Ltd. This is an Open Access article distributed under the terms of the Creative Commons Attribution License (http://creativecommons.org/licenses/by/2.0), which permits unrestricted use, distribution, and reproduction in any medium, provided the original work is properly cited. The Creative Commons Public Domain Dedication waiver (http://creativecommons.org/publicdomain/zero/1.0/) applies to the data made available in this article, unless otherwise stated. 
be a non-vector because its zoophagic [6,7], or at least highly opportunistic [8], host-preference effectively disrupts the human-to-human cycle of transmission required by $P$. falciparum. In contrast, female An. gambiae s.s. are especially efficient at transmitting human diseases because they preferentially obtain blood meals from human hosts, a behavioral trait (anthrophagy) of relatively recent origin $[9,10]$.

Host seeking in mosquitoes is strongly influenced by olfactory and other sensory cues transduced by a variety of proteins that comprise the relevant transduction pathways [11,12]. In mosquitoes, olfactory genes are expressed in and around olfactory receptor neurons (ORNs) that are themselves contained within specialized chemosensory tissues and structures [13-18]. In most arthropods, ORNs are most highly concentrated within the antenna and, in mosquitoes, modulation of antennal ORN physiology has been correlated with some behavioral phenotypes $[19,20]$. The expression patterns of these genes along with the heterologous deorphanization of odor sensitivities of the sensory receptors that are central to these processes have helped refine our understanding of the links between chemosensory driven signaling and behavior [13,15,21-26]. Therefore, there is reason to suspect that species-specific, phenotypic variation between olfactory mediated behaviors may be informed by examining variation displayed by chemosensory genes, in terms of both molecular sequence and transcript abundance [27-30].

Several chemosensory gene families have been identified in An. gambiae, including odorant (Agam $\backslash$ Ors, hereafter referred to as AgOrs), gustatory (Agam $\backslash$ Grs, hereafter referred to as $A g G r s$ ), and variant ionotropic glutamate (Agam \Irs, hereafter referred to as AgIrs) receptors, as well as odorant binding proteins (Agam $\backslash$ Obps hereafter referred to as AgObps) [22,31-33]. These large multigene families encode proteins that are likely to account for the majority of chemical sensitivities in adult peripheral sensory appendages. For example, most AgOrs are transcribed in the antennae [21,27] and transcript abundances of many AgOrs are altered following a bloodmeal [28]. Furthermore, examination of AgOr response profiles in heterologous expression assays has identified numerous compounds from diverse chemical classes that are known activators of ORNs and behavioral attraction [25,26,34]. For example, among the recognized AgOr ligands are components of human sweat that have been implicated in An. gambiae host-seeking [35-38]. These alterations in AgOr transcript abundance in response to specific cues lead to apparent shifts in the potential receptivity of female antennae, including an enhancement of the response to 2-propylphenol, a compound that can act as an oviposition stimulant [28].

Most AgGrs, like their D. melanogaster counterparts, are assumed to encode receptors for sweet and bitter compounds as well as for other tastants [22,39]. However, transcripts for a small number of AgGrs are also enhanced in adult antennae where they may function in volatile chemical reception [27]. Moreover, three of the AgGrs encode palp-expressed receptors for carbon dioxide, an important activator of upwind flight in female mosquitoes $[7,23,40]$.

While the AgIrs have not been fully characterized, many members of this gene family are expressed in adult appendages [27]. Based on their functions in An. gambiae larvae [32] and homologies to D. melanogaster DmIrs [33,41], the AgIrs are potential receptors for amines and acids which comprise host kairomones whose ORN sensitivities are housed in the basiconic, or grooved peg, antennal sensilla [19]. Several AgIrs show reduced transcript abundance following a bloodmeal, leading some to hypothesize this chemoreceptor family may also contribute to the observed host-seeking refractoriness in recently-bloodfed females [28].

$A g O b p$ transcripts are broadly present in adult head appendages at very high levels $[16,27,42]$. In addition, transcripts for many AgObps are specifically enhanced in body tissues, where their function remains uncharacterized. Nonetheless, knockdowns of antennal-expressed Obp1 in mosquitoes leads to impaired indole responsiveness and indicates that OBPs function in mosquito peripheral odor sensitivities $[43,44]$.

To test whether phenotypic host preference may be associated with the peripheral expression profiles of chemosensory genes such as chemoreceptors and Obps, we have isolated and sequenced mRNA from the antennae of An. gambiae and An. quadriannulatus to compare their transcriptome profiles in non-blood fed, female mosquitoes. We found that while there were high levels of similarity in the type and number of chemosensory genes detectable in the antennae of both species, there were significant divergences at both the molecular and transcriptional levels. Furthermore, differences in the antennal chemoreceptor composition, most notably within the $O R$ family, appeared to express a subset of the An. quadriannulatus chemosensory repertoire within An. gambiae, that may be particularly refined for the detection of human associated host cues.

\section{Results and discussion}

An. gambiae and An. quadriannulatus share highly similar chemosensory gene repertoires

We first compared the number of chemosensory genes in the genomes of An. gambiae and An. quadriannulatus. While the size and composition of An. gambiae chemosensory gene families have been reported previously [22,27,31], little is known about An. quadriannulatus since its genome sequence has only recently become publically available (https://olive.broadinstitute.org/projects/anopheles). 
To fully characterize the chemosensory repertoire in An. quadriannulatus, we conducted exhaustive and iterative searches for homologs of known insect chemosensory genes using a rigorous pipeline and carefully annotated gene models [45] (see Materials and Methods). The same procedure was also applied on An. gambiae to eliminate potential bias introduced by gene annotation. In total, we identified 74 ORs, 60 GRs, 43 IRs, and 75 OBPs in $A n$. quadriannulatus, as well as 75 ORs, 61 GRs, 46 IRs, and 80 OBPs in An. gambiae (Figure 1).

Comparison of the An. gambiae and An. quadriannulatus annotations revealed the presence of a relatively stable overall number of chemosensory genes in the two species, which suggests that their repertoires are fairly conserved; although we cannot entirely rule out the hypothesis of rapid underlying gene turnover despite that the total gene number has remained unchanged. To distinguish between these two scenarios, we further investigated the evolutionary dynamics of chemosensory genes in An. gambiae and An. quadriannulatus. Based upon our phylogenetic analysis (Additional file 1: Dataset S1), chemosensory genes can be classified into 253 orthologous groups (OGs), including 75 OGs of ORs, 61 OGs of GRs, 43 OGs of IRs, and 74 OGs of OBPs (Figure 1), where each OG represents a single gene in the most recent common ancestor (MRCA) of the two species. In this view, the estimated number of chemosensory genes in the MRCA is nearly identical to the number we observe in the two present species (Figure 1). Furthermore, the vast majority of OGs are comprised of one gene from each species; only 12 of the 253 OGs show evidence for one or two gene gain or loss events (Figure 1). Taken together, these data support the hypothesis that the chemosensory gene repertoire has been stable following the speciation of An. gambiae and An. quadriannulatus.

Rapid gene birth-and-death is a signature feature of the molecular evolution of insect chemosensory genes [46], as revealed by comparisons of species with various levels of relatedness and varying reliance on chemosensation. Lineage-specific variations in the size of chemosensory families are usually correlated with altered requirements on chemosensation posed by changes in life style and ecology $[47,48]$. As shown in the comparative studies of generalist and specialist sister species in Drosophila, D. sechellia likely underwent dramatic host specialization after its divergence from the generalist D. simulans [49-52]. This behavioral change was accompanied by both an accelerated rate of gene loss and an elevated level of sequence divergence of chemosensory receptors of $D$. sechellia, likely reflecting a reduced, but more focused chemosensory capability due to the more restricted host range associated with geographic isolation [49-52].

In contrast, our comparison of the four chemosensory families between the anthropophilic mosquito An. gambiae and its zoophilic sibling An. quadriannulatus, revealed only slight variations in gene number. Indeed, the two species differ by only one gene in both $O R$ and $G R$ families although these two types of receptors are vital for mosquito host seeking and preference. Among other chemosensory genes, the three $A n$. gambiae specific IR duplicates belong to the subfamily of "divergent IRs" which are most abundant outside antennae $[27,32,33]$; there is a paucity of functional information for the OBPs that have been duplicated or lost, thus a rationale for these events remains elusive.

This discrepancy in the evolutionary pattern could be due to several factors. Firstly, the divergence of An. gambiae and $A n$. quadriannulatus is estimated to have occurred very recently, as little as only several thousand years ago $[10,53]$, coinciding with the increased availability of human hosts that paralleled the growth of agriculturally-based communities; this is significantly less than the $\sim 0.5$ million years separation of $D$. sechellia and D. simulans [54]. Our results suggest that different modes of chemosensory gene evolution have played major roles at different time-scales; genomic changes at levels other than gene copy number

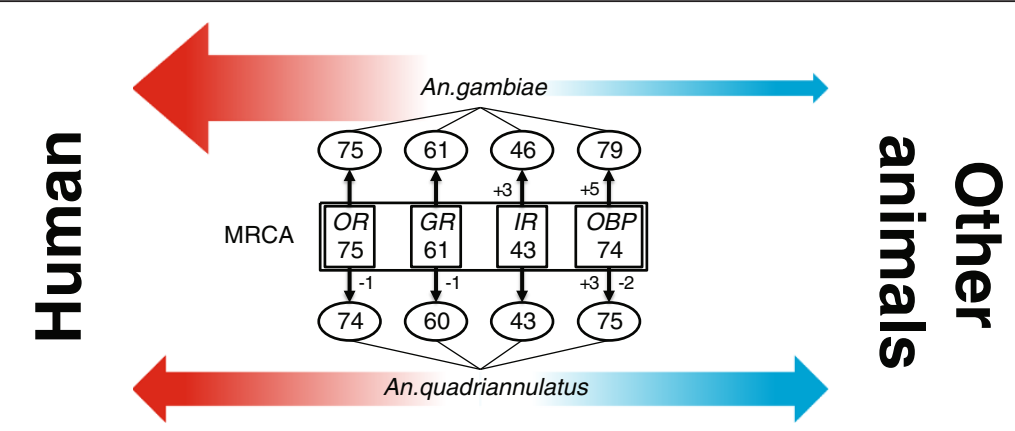

Figure 1 Chemosensory gene repertoires of An. gambiae and An. quadriannulatus. The numbers of An. gambiae and An. quadriannulatus chemosensory genes annotated in this study (ovals). The estimated numbers of chemosensory genes in the most recent common ancestor (MRCA) of the two species (boxes). The numbers along vertical arrows indicate the estimated numbers of gene gain (+) and loss $(-)$ events. The red and blue schematic arrows indicate the host preferences (red: anthropophagic, blue: zoophagic) of An. gambiae and An. quadriannulatus. 
are likely to have rapidly driven the behavioral divergence between the two mosquitoes over a very short period of time. Moreover, the zoophagy of An. quadriannulatus likely represents the ancestral state and $A n$. gambiae acquired the preference for humans later [9]. It has been suggested for phytophagous insects that the specialization to a fraction of its ancestral host range usually involves altered sensitivity to odors for both previous and new hosts (for preference) [55]. Similarly, the adaptation of $A n$. gambiae to human hosts may have required more acute responses to both attractants of human origin and deterrents of animal origin in comparison to An. quadriannulatus. Such differences could have been achieved either by functional divergence or by differential expression/abundance of orthologous chemosensory genes between the two mosquitoes, or both.

\section{Chemosensory genes underwent rapid sequence evolution}

Our overall comparison of chemosensory genes between An. gambiae and An. quadriannulatus raises the possibility that, given the largely shared repertoire, the functional divergence between orthologs may be an important factor underlying the shift in host preference. That said, the lack of any structural insight and functional data for most chemosensory genes hinders a direct comparison of ligand sensitivities between orthologous genes. However, the role of functional divergence can still be assessed in part by examining the pattern of chemosensory gene evolution at the sequence level. To begin to address this, we investigated the evolution of each of the 241 one-to-one orthologous pairs of chemosensory genes by using two metrics: (1) the rate of amino acid substitution (protein distance), which represents the rate of protein sequence divergence; and (2) the ratio of non-synonymous substitution rate to synonymous substitution rate $(\mathrm{dN} / \mathrm{dS}$ ratio), which estimates the influence of natural selection on protein coding sequences (Additional file 2, Table S1).

As shown in Figure 2, while there are considerable variations in evolutionary rates among chemosensory genes, all four chemosensory families have significantly higher median values of protein distance and $\mathrm{dN} / \mathrm{dS}$ ratio as compared to other genes, suggesting that chemosensory genes as a whole evolved more rapidly than their respective transcriptome backgrounds. Among gene families, the IRs display the highest median values of both measurements, mostly driven by the "divergent IRs", followed by ORs and GRs. While OBPs appear to have somewhat overall lower evolutionary rates, some of the most rapidly evolving chemosensory genes are also found in this family. Within each family, genes are broadly distributed across the range of protein distance and $\mathrm{dN} / \mathrm{dS}$ ratios. While genes encoding OR and IR co-receptors and GR carbon dioxide receptors show extremely low evolutionary rates, there are 3 genes with $\mathrm{dN} / \mathrm{dS}$ ratios $>1$ (Gr3, Ir139, and Obp15), and a number of others with $\mathrm{dN} / \mathrm{dS}$ ratios around 0.5 . While large $\mathrm{dN} / \mathrm{dS}$ ratios $(>1)$ are considered to be evidence for positive selection, intermediate values may indicate relaxed purifying selection, or they could reflect positive selection on a fraction of the gene sequence.

These two measurements of evolutionary rate show an overall positive correlation in all four chemosensory families (Additional file 3: Figure S1). However, there are also multiple examples where orthologous gene pairs display high $\mathrm{dN} / \mathrm{dS}$ ratios but only a small number of amino acid changes (e.g. Or24, Or36, Gr3, Gr57, and Ir141). These genes are most likely the result of positive selection; while both positive selection and relaxed purifying selection can lead to elevated $\mathrm{dN} / \mathrm{dS}$ ratios, genes under relaxed purifying selection would also be expected to have a higher rate of amino acid substitution than is seen here.

Genes under both types of selection represent potential candidates for genomic determinants of the behavioral and electrophysiological response differences between An. gambiae and An. quadriannulatus. Differential odor responses that are mediated by functional divergence of chemosensory genes would most likely require positive selection on genes that are responsible for the detection of human attractants and/or non-human deterrents, leading to increased sensitivity for these semiochemicals. On the other hand, receptors whose ligands include human deterrents and non-human attractants would possibly experience relaxed selective constraints as amino acid changes that attenuate these responses would be less deleterious or even beneficial. To look for additional evidence of functional divergence, we characterized the rate of conservative and radical amino acid substitutions and the distribution of these substitutions on the primary sequences of OR proteins. In contrast to conservative and typically neutral substitutions, radical amino acid substitutions are more likely to alter protein function; therefore the ratio of radical substitution rate to conservative substitution rate $(\mathrm{dR} / \mathrm{dC})$ is also a very useful measurement of selective pressure on protein evolution [56,57]. Using this metric, we identified $\mathrm{dR} / \mathrm{dC}$ ratios $>1$ for 6 Ors, 12 Grs, 4 Irs, and 3 Obps (Additional file 2: Table S1), suggesting these genes might also be under positive selection.

Insect $O R$ genes encode 7 transmembrane (TM) proteins and at least one previous study has suggested that TM domains in OR proteins participate in receptor-ligand interaction [58]. On that basis, we performed topology predictions for all $A g / A q O r s$ and counted conservative and radical substitutions specifically within the predicted TM domain regions. In total, 56 out of 71 ORs have one or more amino acid substitutions in TM domains, including at least one radical substitution in 43 OR genes (Additional file 4: Figure S2 and Additional file 5: Table S2). Inasmuch as negatively charged amino acids such as 

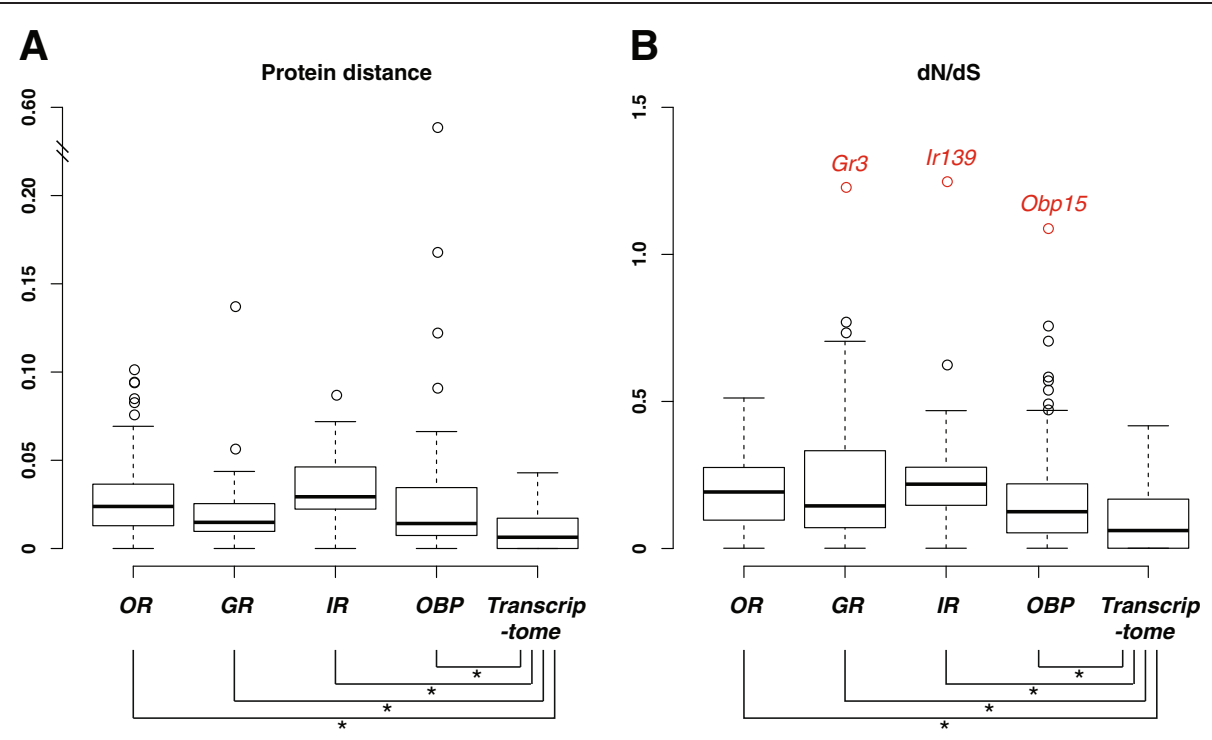

Figure 2 Evolutionary rates of anopheline chemosensory genes. Box plots of (A) protein distances and (B) dN/dS ratios between orthologous pairs of $O R, G R, I R$, and $O B P$ genes. Orthologous gene pairs delineated from An. gambiae genome and An. quadriannulatus transcriptome assembly were used as control. Wilcoxon rank sum tests showed that evolutionary rates were significantly different between chemosensory gene families and the control (* denotes $p$-value $<0.001$ ). Outliers (circles) are shown for chemosensory gene families but not for the transcriptome background due to their large numbers. Ortholog pairs having a dN/dS value greater than one are noted in red.

glutamic acid, asparagine, and tyrosine are involved in defining OR function [59], the frequency of replacements targeting these specific residues was also assessed. Indeed, 38 out of 71 OR proteins contain at least one substitution of a negatively charged residue and 6 ORs manifest these substitutions within predicted TM domains. Taken together, our comprehensive sequence analyses of chemosensory genes have identified multiple types of alterations that suggest that some degree of functional divergence may have occurred between these closely related sibling species of anophelines.

\section{Chemosensory genes are differentially abundant between the two species}

To address the contribution of changes in chemosensory gene transcript levels to the behavioral differences between An. gambiae and An. quadriannulatus, we compared the antennal transcriptome profiles of the two species, focusing specifically on the differential enrichment of chemosensory genes in each of the $O B P, I R$, and $O R$ families (the $G R$ family was not discussed here due to the lack of meaningful antennal expression). Among the transcripts detected in the antenna of An. quadriannulatus, our findings were broadly consistent with previous RNAseq studies in An. gambiae [27,28], and both species showed extensive conservation in the number and identity of detectable, chemosensory genes (Additional file 6: Table S3). Indeed, we only identified a few instances of species-specific chemoreceptor expression; the most notable occurrences were Or33 in An. quadriannulatus and Ir7s in An. gambiae which both displayed transcript abundance levels above the median level for all transcripts. Such profound overlap in the variety of expressed, chemosensory genes may not be surprising given the level of genomic conservation and serves to reinforce the evolutionary proximity of these two species.

The most abundant chemosensory gene family in the mosquito antenna was the OBPs. The antennal OBPs in both $A n$. gambiae and An. quadriannulatus belonged exclusively to the "classical" subclass of $O B P$, an observation consistent with our previous study that detected "atypical" AgObps in the antennae at only a single, discreet time point following a bloodmeal [28]. All OBP transcripts were much more abundant in the antennae of An. gambiae, with the total RPKM of detectable OBPs nearly twice that for the $O B P s$ found in An. quadriannulatus. Indeed, the OBPs were the only family of chemosensory genes that was overrepresented in An. gambiae, with every detectable $O B P$ displaying a significant difference in transcript abundance (Figure 3). Interestingly, despite the nearly 2:1 disparity in gross, $O B P$ transcript abundance, the expression-based rank order of OBPs remained highly conserved between the two species (Spearman's $r=0.94$ ), and was more highly correlated than that of either the IRs $(\mathrm{r}=0.70)$ or the ORs $(\mathrm{r}=0.64)$. While on the whole, the $O B P$ gene family plays diverse roles in insects, the similar presence and distribution of this distinct subset of OBPs between An. gambiae and An. quadriannulatus 

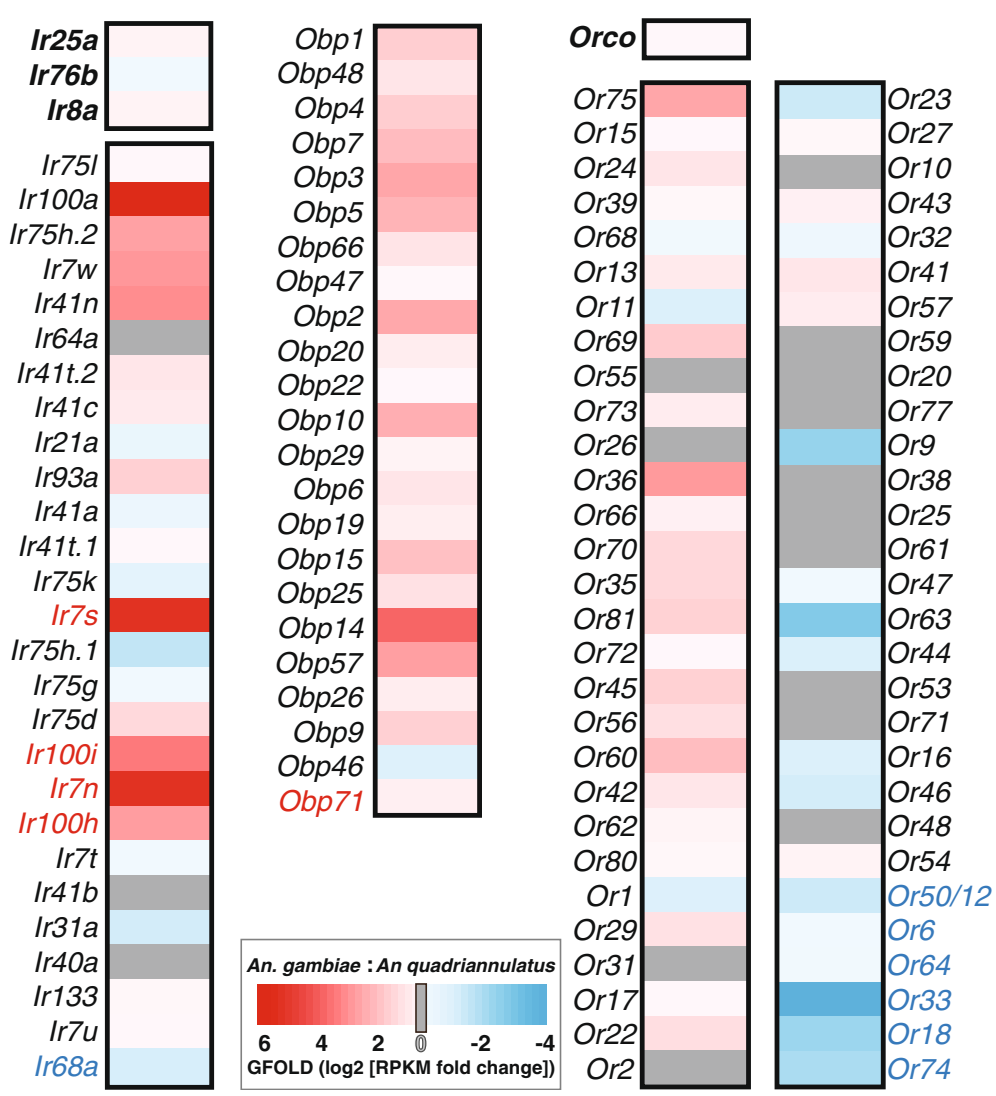

Figure 3 Differential antennal abundances of chemosensory genes. Heat maps for the three most abundant chemoreceptor gene families in the antenna. Only transcripts that were detectable one or both species are displayed. Colors indicate a normalized, GFOLD score (reliable log2 fold difference in transcript abundance) denoting enrichment in either An. gambiae (red) or An. quadriannulatus (blue); genes showing no discernible expression differences between the species (GFOLD=0) are shown in grey. The gene names are ordered (from high to low, top to bottom) based upon their relative abundance in An. gambiae. Co-receptors are displayed in bold above the $O R$ and $I R$ gene family heat maps. Chemoreceptor names are color coded if they were only classified as being detectable in An. gambiae (red) or in An. quadriannulatus (blue).

suggests they are more conserved within anopheline olfactory tissues, relative to the evolutionarily labile membrane bound, ligand specific chemoreceptors.

In contrast to the OBPs, the IRs and ORs exhibited widespread variation in transcript abundances between An. gambiae and An. quadriannulatus (Figure 3). The antennal IRs displayed the most instances of transcript variation, with 27 of the 30 detectable IRs showing significant differences in abundance. Moreover, the sum total of the presumptive $I R$ co-receptors (i.e., the combined RPKM values for $\operatorname{Ir} 25 a, \operatorname{Ir} 76 b$ and $\operatorname{Ir} 8 a$ ) is more than $60 \%$ higher in $A n$. quadriannulatus. This divergence in $I R$ expression levels, in conjunction with the low coefficient of determination in the rank order of IRs between $A n$. gambiae and An. quadriannulatus $\left(\mathrm{r}^{2}=0.49\right)$, distinguishes the IRs as the most variable chemosensory gene family between the sibling species. This greater variability parallels the molecular evolutionary analysis above, which showed the IR family to display the highest degree of sequence divergence among the chemo-receptors
(Figure 2). Because the ultimate roles and functions of the individual members of $I R$ family are still being defined, the precise impact of these observed differences is as yet unclear. However, the pervasive, interspecific variation in both sequence composition and transcript abundance may indicate that IRs are especially adaptable. This represents a chemoreceptor class that may be involved in both mediating internal signals as well as sensing external environmental cues.

The ORs are the best characterized class of chemoreceptor in insects. Many functional aspects of dipteran ORs have been determined for both $D$. melanogaster and An. gambiae, and the results consistently show that individual Dm- and AgOrs display their own distinct range of odor selectivity or "tuning" [25,26]. For example a recent report suggests that $O R s$ as a class are critical to defining mosquito host-specificity [60].

ORs are localized in the dendritic membranes of ORNs and require the presence of a conserved odorant receptor co-receptor (Orco) for correct localization and subsequent 
function [17,61]. Because Orco is always required for $O R$ function, its abundance may be taken as a general proxy for overall $O R$ abundance. By that measure, An. gambiae antennae displayed only a modest (6\%) enrichment in the Orco transcript abundance compared with $A n$. quadriannulatus, and we can reasonably conclude that the overall expression levels of ORs are consistent between the two species. Indeed, this conservation is in keeping with previous, comparative morphological studies that reported a slightly higher density of sensilla on $A n$. gambiae antenna, including the highly abundant trichoid sensilla [62] that house three Orco-expressing ORNs $[13,16]$. Therefore, while An. gambiae antennae might possess a very slight advantage in OR-mediated odor sensitivity, our transcriptional data largely agrees with the comparative morphologic study to imply that that both species share equivalent olfactory capabilities [62].

Similarly, in both species half of the sum totals of tuning $O R$ transcripts in the antenna were comprised of a small, largely identical subset of either 7 ORs in An. gambiae or 8 ORs in An. quadriannulatus. Within this top 50\%, 5 ORs were shared between species (Ors 11, 15, 24, 68 and 75) and had an average $\mathrm{dN} / \mathrm{dS}$ below that of the $O R$ class as a whole. Therefore, in terms of relative transcript abundance, most of the predominant antennal Ors shared between the species were also more conserved at the sequence level.

Beyond these similarities, the composition of the remainder of the tuning $O R$ pool appeared to vary substantially between the two species (Figure 3). In total, 49 of 58 (84\%) tuning ORs showed significant differences, 16 of which were more than a 2-fold enriched in one of the species.

In An. gambiae antennae, the most noticeable overall trend in differential $O R$ abundance was the degree to which select ORs were enriched as compared to $A n$. quadriannulatus (Figure 4). While there were no ORs whose antennal expression appeared specific to An. gambiae,
29 tuning ORs showed significant levels of enrichment in An. gambiae, with ORs 36, 60, 69, and 75 each showing as much as a 4-6 fold enrichment (Figure 3). Overall, these An. gambiae enriched ORs were 6-fold more abundant than the combined pool of depleted ORs. This stands in marked contrast to the balanced distribution of ORs in An. quadriannulatus, with enriched and depleted ORs showing similar expression levels in terms of overall RPKM (Figure 4). Taken together, the OR-mediated odor coding of the An. gambiae antennae appears to be an overrepresented subset (Fisher's Exact test, $p=2.2 \times 10^{-16}$ ) of ORs whose orthologs are also present in An. quadriannulatus. This sizeable skew in the distribution of ORs implies that the An. gambiae antenna predominantly expresses only a subset of those ORs within the antenna of An. quadriannulatus.

When differential levels of $O R$ transcripts were viewed within the context of molecular divergence (Figure 5), there was no significant correlation between transcript enrichment and $\mathrm{dN} / \mathrm{dS}$ ratio. However, it was clear that ORs with higher evolutionary rates were also more variable in terms of transcript enrichment and tended to display higher enrichment levels. When ORs were analyzed in quartiles based on their $\mathrm{dN} / \mathrm{dS}$ ratios, the upper three quartiles $(\mathrm{dN} / \mathrm{dS}$ ratio $\geq 0.1)$ showed significantly higher median and variance values of transcript enrichment as compared with the first quartile, either individually or collectively (see Additional file 7: Table S4). Interestingly, the opposite trend was observed at the level of the antennal transcriptome profile, where genes in the first quartile (with lower dN/dS ratios) displayed greater magnitude and variability of transcript enrichment (see Additional file 7: Table S4). In addition, ORs with $\mathrm{dN} / \mathrm{dS}$ ratios above the transcriptome median $(0.0611)$ comprised the majority of detectable ORs and showed significantly higher levels of enrichment than those genes

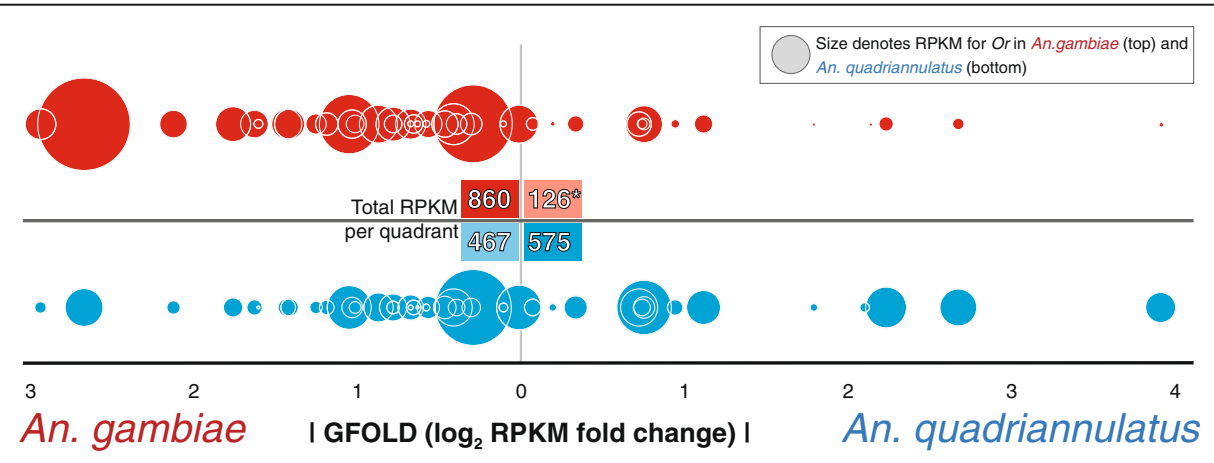

Figure 4 Distribution of differentially abundant antennal Ors and their relative abundance levels in An. gambiae and An. quadriannulatus. Individual tuning Or orthologs are represented by bubbles with areas scaled to their respective abundance (RPKM) in either An. gambiae (red) or An. quadriannulatus (blue). Or orthologs are arranged horizontally based upon their enrichment (GFOLD value) in either An. gambiae (left) or An. quadriannulatus (right). Total RPKMs for each quadrant are indicated in the center. The asterisk denotes the larger than expected proportion of Or abundance in An. gambiae ascribable to Ors that are also enriched in An. gambiae (Fisher's Exact Test, $p=2.2 \times 10^{-16}$ ). 


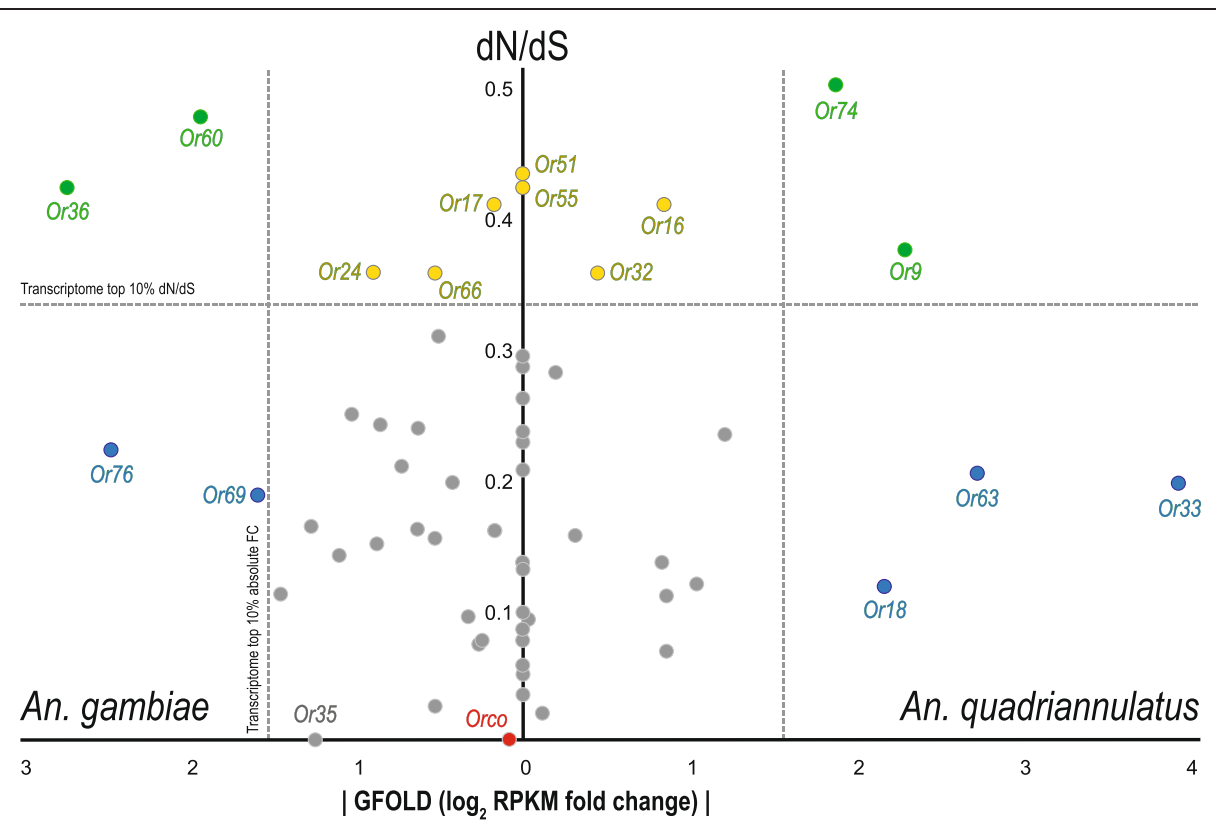

Figure 5 Differential expression of antennal ORs plotted against dN/dS. X-axis represents the absolute GFOLD score (reliable log2 fold difference in transcript abundance) for Ors enriched in either An. gambiae (left half) or in An. quadriannulatus (right half). Ors displaying no significant difference in transcript abundance are plotted at zero. Y-axis is the interspecific dN/dS for each Or. Ors are color coded as follows: grey: conserved in sequence and in transcription, blue: conserved in sequence but diverged in transcription, yellow: diverged in sequence but conserved in transcription, green: diverged in sequence and in transcription. Horizontal dashed line denotes the top 10\% of transcriptome wide $\mathrm{dN} / \mathrm{dS}$ value and the vertical dashed line denotes the top $10 \%$ of transcriptome wide, absolute fold change.

in the transcriptome background in the upper half of the $\mathrm{dN} / \mathrm{dS}$ (Wilcoxon rank sum test, $\mathrm{p}=0.02792$ ). This contrast, once again, highlights that $O R$ s are under rapid evolution at both sequence and expression levels.

Overall, there were 11 and 9 ORs that resided in the top $10 \%$ of the transcriptome profile in terms of their evolutionary rates and absolute levels of transcript enrichment, respectively (Figure 5). Four of these ORs showed both high sequence divergence and abundance differences, while the remaining genes differed in either sequence or abundance. This pattern suggests that sequence divergence and differential abundance represent two non-mutually exclusive mechanisms for the evolution of ORs, and perhaps other chemosensory genes. Those ORs with exceptionally high levels of sequence divergence and/or transcript enrichment likely play important roles in chemosensory-mediated behavioral differences between An. gambiae and An. quadriannulatus. Some of the relatively more conserved ORs might be interesting as well. For instance, Or35 is the most conserved tuning $O R$ but its absolute fold change was ranked within the top $20 \%$ of the antennal transcriptome profile.

\section{Differential receptivity analysis}

We have previously integrated $O R$ functional data with RNAseq data to model the receptivity profile for the antenna of An. gambiae following a bloodmeal [28]. This analytical approach synthesized the effects of many small changes in the expression profiles of individual tuning ORs to treat the antenna as a single, chemosensory unit. Applying the same methodology here, to effectively map the An. gambiae odorant receptivity onto the An. quadriannulatus OR transcriptome profile, we modeled potential odor-coding differences between these two species. While it is important to note that this approach assumes the general functional conservation among interspecific $O R$ orthologs, this is a reasonable assumption given that non-conservative substitutions observed among the ORs occur in the trans-membrane and intra-cellular loop regions and are therefore most likely to impact the channel properties of the Orco-OR complex rather than OR-ligand interactions [59].

While the results of this analysis showed the species to share a similar level of receptivity toward three floral compounds (fenchone, isobutyl-acetate and methyl-benzoate), there appears to be a general reduction in relative receptivity within the An. gambiae antenna to many of the odorants tested. An. quadriannulatus appeared more receptive to a wide range of chemical classes including most aromatic compounds and many alcohols (Figure 6; Additional file 8: Table S5), and while many of these compounds are plant associated some are also components of human skin [36,38,63-65]. Of those compounds to which An. quadriannulatus appears more 


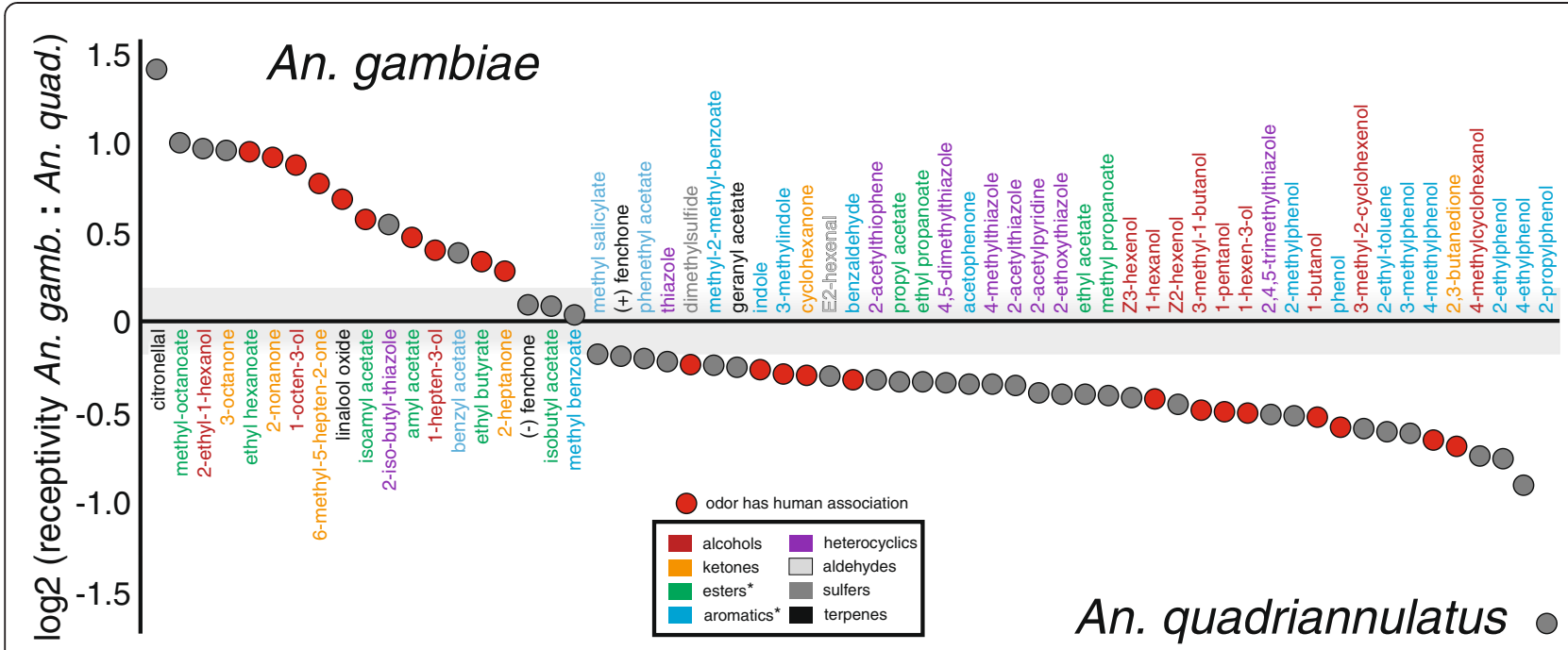

Figure 6 Differences in $O R$ mediated odorant receptivity between An. gambiae and An. quadriannulatus antennae. Vertical axis represents computed, interspecific differences in antennal receptivity to a panel of odors. Displayed results are sorted left to right based upon the level of each odor's relative receptivity enhancement in either An. gambiae (positive values) or An. quadriannulatus (negative values). The grey region around zero denotes an absolute change in relative receptivity of $10 \%$ or less. Chemical names are color coded by chemical class and asterisks denote chemical classes whose receptivity is disproportionately represented in one species (Fishers Exact Test, $p<0.05$ ). Red points denote odors that have been detected in human-associated skin emanations.

receptive, the two indolic compounds are known to be important to the chemical ecology of many mosquito species [36,37,64,66-68]. While both indole and 3-methylindole have been characterized as human associated compounds $[36,64,69]$, they are also associated with other natural sources, including decaying organic material and animal excreta [66]. Accordingly, we cannot discount the possibility that the same odorant can elicit different perceptions dependent upon ecological context. Nevertheless, the presence of these compounds along with the several other human associated odorants can also be rationalized within the context of human host-seeking since $A n$. quadriannulatus displays limited, anthropophagic behavior as well [70].

In contrast, the $O R$-mediated olfactory specialization of An. gambiae antenna appears to be heavily biased (Fishers Exact test, $p=0.06$ ) toward odors which have been previously associated with human skin emanation, including a majority of the esters assayed (Fishers Exact test, $p=0.04$ ). Furthermore, if we only consider compounds that showed a change in relative receptivity greater than $10 \%$ in either species that show only minimal, the apparent enhanced receptivity of $A n$. gambiae to humanassociated odor chemicals becomes even more significant (Fisher's Exact test $\mathrm{p}=0.02$ ). Moreover, some human associated odors have greater magnitudes of receptivity enhancement in An. gambiae to as compared to any of those in An. quadriannulatus (Figure 6). This notable trend agrees with both the molecular and the transcriptional analyses above, further suggesting that at the molecular level, the OR-mediated sensitivity of the antennae of $A n$. gambiae appears to be more focused and specialized than that of $A n$. quadriannulatus.

\section{Conclusions}

In this study we examined the RNA composition of the peripheral chemosensory tissues of An. gambiae s.s. and An. quadriannulatus, two closely related members of the An. gambiae species complex. Because these two species are phenotypically divergent in terms of their host seeking predilections, we looked specifically at differences within the chemosensory gene classes, both at the molecular level and at the transcriptional level. Overall, while the chemosensory gene repertoire was highly conserved, we found that rates of evolution of each of the chemosensory gene families were more rapid than the genomic background. In particular, we identified considerable levels of radical amino acid changes between orthologous $O R$ genes that may potentially result in functional differences. To our knowledge, this is the first comparative study of the chemosensory gene repertoire between sibling species that are diverged by only several thousand years ago. Unlike the dramatic copy number changes often observed in comparisons of more distantly related species, these results suggest that functional divergence between orthologous chemosensory genes may be key in driving behavioral differences in the immediate aftermath of speciation events.

A careful analysis of their antennal transcriptome profiles also revealed both the overall conservation of some critical 
chemosensory transcripts (e.g. Orco), along with large degrees of abundance differences among some individual gene family members. The observed similarities confirm results of prior morphological studies that reported the antennae of both species share similar sensilla densities overall [62]. Though no ORs appeared to be exclusively expressed within the An. gambiae antenna, the divergence in the overall transcriptional profile of the ORs was considerable. The specific ORs whose transcripts comprise the preponderance of $O R$ transcripts within the antennae of An. gambiae are also greatly enriched as compared to $A n$. quadriannulatus, indicating that in terms of $O R$ composition, the An. gambiae antenna appears most likely to be a specialization of the An. quadriannulatus antenna.

When these interspecific abundance differences in the $O R$ gene family members were integrated in silico with AgOr functional data, the resulting antennal "receptivities" again indicated that the human-biased odor receptivity of An. gambiae was most likely a refinement of that of An. quadriannulatus. Moreover, this biased receptivity of An. gambiae antenna toward human-derived odors may be further augmented by the functional differences between orthologous ORs suggested by our sequence analyses. Future functional tests of $\mathrm{AqOr}$-odor tuning will further improve our understanding in this regard.

Taken together, and given the central role that ORs play in defining host specificity, the anthropophagy of $A n$. gambiae is most likely not derived from the evolution of any single $O R$ specific for the purpose of human host seeking. Instead, we posit the receptivity bias in the antenna of An. gambiae toward human host odors is likely the result of the cumulative effects of both functional divergences and changes in the abundance and distribution of common ORs already present within the An. gambiae species complex.

\section{Methods}

\section{Gene annotation}

The genome assemblies of An. gambiae (version AgamP3) and An. quadriannulatus (version 1) were downloaded from the websites of VectorBase (www.vectorbase.org) and Broad Institute (olive.broadinstitute.org), respectively. The annotation of chemosensory genes was performed following a previous protocol [45]. In brief, previously reported chemosensory genes from An. gambiae, Aedes aegypti, Culex quinquefasciatus, and D. melanogaster were used as queries in TBLASTN [71] searches against the two anopheline genomes. Putative chemosensory gene coding loci were identified after filtering out low-scoring blast hits. For each locus, the query sequence that yield the highest bit score was selected as reference to perform homology-based gene prediction using GeneWise (version 2.2.0; [72]). All gene models were manually inspected and modified if needed. All genomic data is available through VectorBase and the annotated chemoreceptor sequences are listed in supplementary Table S1.

\section{Phylogenetic analysis}

For each of the $O R / G R / I R / O B P$ families, protein sequences of genes in the two mosquitoes were aligned using MAFFT (version 7.037b; [73]). The multiple sequence alignments were manually curated and poorly aligned regions were removed using trimAl (version 1.4; [74]) with "automated1" option. Maximum-likelihood trees were constructed using RAxML (version 7.4.7; [75]) and the reliability of tree topology was evaluated with 100 bootstrap replicates. Resulting gene trees were reconciled with the species phylogeny to estimate ancestral gene copy numbers and gene gain and loss events. An orthologous group is defined as a highly supported clade (greater than 90\%) representing a single gene in the common ancestor of An. gambiae and An. quadriannulatus.

\section{Analysis of sequence divergence}

For each orthologous pair of chemosensory genes in An. gambiae and An. quadriannulatus, protein sequences were aligned using MAFFT and the corresponding nucleotide alignment was generated using a custom script (available upon request). The rate of amino acid substitution and $\mathrm{dN} / \mathrm{dS}$ ratio were calculated using PROTDIST (from the Phylip package version 3.69) and CodeML (from the PAML package version 4.7; [76]), respectively. The $\mathrm{dR} / \mathrm{dC}$ ratio was calculated using the Zhang method [77], for which radical and conservative amino acid changes were defined by the Dayhoff classes ("AGPST", "DENQ", "HKR", "ILMV", "FWY", and "C"). The topologies of Or proteins were predicted using TOPCONS [78] and the number of radical/conservative amino acid changes in transmembrane domain regions were counted accordingly.

To identify additional orthologous gene pairs between the two mosquitoes, de novo transcriptome assembly of An. quadriannulatus was generated and likely coding regions were extracted, both using Trinity (version 2012-10-05; [79]) Orthologous groups were then constructed from annotated genes in An. gambiae (version AgamP3.7) and likely coding sequences in An. quadriannulatus using orthoMCL (version 2.0.5; [80]) Protein divergence, $\mathrm{dN} / \mathrm{dS}$ ratio, and $\mathrm{dR} / \mathrm{dC}$ ratio were calculated for each 1-to-1 orthologous pair similarly to chemosensory gene pairs.

\section{Mosquitos and mosquito rearing}

An. gambiae sensu stricto (SUA 2La/2La, an M-form isolate originating from Suakoko, Liberia) and $A n$. quadriannulatus (SKUQUA, an A form isolate originating 
from Skukuza, South Africa) were reared in the Vanderbilt Insectary Facility as described previously [21]. Adult mosquitoes were reared under 12:12 light-dark conditions and had constant access to $10 \%$ sucrose solution.

\section{RNA isolation and RNA sequencing}

Four to six day old adult female mosquitoes from each species were collected in the middle of the light phase ( ZT6) for antennal resection. For each collection, antennae were hand-resected into TRIzol, and total RNA was isolated. mRNA isolation and cDNA library preparation were carried out using the Illumina mRNA sequencing kit (Illumina Inc; San Diego, CA). Libraries were barcoded and sequenced in paired-end fashion (50PE An. quadriannulatus, 100PE for $A n$. gambiae) on an Illumina HiSeq2000. Approximately 30 million reads were generated for each sample. No biological replicates were preformed becasue sampleto-sample variation in RNAseq results among anophelene antennae has been observed to be very low (Additional file 9: Figure S3).

\section{Data processing and abundance profiling}

Individual Illumina read files (fastq) were trimmed and filtered using Trimmomatic, a software package specifically designed for trimming NGS reads. Paired end Trimmomatic parameters used were: LEADING:3 TRAILING:3 SLIDINGWINDOW:4:15 MINLEN:36. FastQC was used for data set quality checking.

To better quantify transcript abundances in An. quadriannulatus, a modified version of the An. gambiae reference genome was prepared to eliminate potential bias caused by genomic sequence differences between the two species. The reads of An. quadriannulatus were first mapped to the An. gambiae reference genome (version AgamP3) using Tophat2 (version 2.0.8) with the guidance of gene annotation (version AgamP3.7), and only one alignment was reported for each mapped read. Fixed differences between the species were called and filtered using SAMtools (version 0.1.18) with a minimum read depth of 5 and variant quality score of 60 . We then replaced nucleotides in the An. gambiae reference genome at sites of fixed differences with each site's most frequent, alternative allele. This modified reference genome sequence was used for subsequent analyses of An. quadriannulatus transcriptome. Finally, reads were then aligned to the respective, indexed genome using Tophat2 [81].

\section{Differential transcript abundance calculation}

Statistical significance along with fold change was determined by pairwise comparison of the Tophat 2 alignments for each of the two species using GFOLD (version 1.0.9 [82]) configured for a 99 percent confidence interval. The result was a set of GFOLD values (a.k.a. GFOLD's "reliable" log2 fold change) for each An. gambiae gene identifier (AGAP); GFOLD values other than zero are considered as significantly, differentially expressed.

\section{Odorant receptivity changes}

Relative differences in odorant receptivity between the An. gambiae and An. quadriannulatus were calculated from physiologic, odorant-response data from previously published functional deorphanization of $A n$. gambiae odorant receptors $[25,26]$. The SSR data was first filtered to remove any Ors or chemicals that failed to elicit a 100 spikes/second increase over baseline in at least one assay. One hundred spikes per second was chosen to retain only more-robustly responding receptors and ligands in an attempt to mitigate any small potency differences that might exist between the species. Odor-induced decreases in spiking frequency were treated as indeterminate and treated as zero. The response of each $\mathrm{AgOr}$ (spikes/second increase) to each odorant was then weighted by the normalized abundance level (RPKM [83]) of that Or. Odorant responses in weighted-spikes-per-second were then summed for each odorant in each species, resulting in an "antennal receptivity" for that species. Finally, the interspecific "receptivity change" of the antenna was calculated by dividing the "antennal receptivity" of An. gambiae by that of An. quadriannulatus.

\section{Additional files}

Additional file 1: A text file includes maximum-likelihood trees of the $O R, G R, I R$, and OBP genes in An. gambiae and An. quadriannulatus.

Additional file 2: A table listing the evolutionary rates for each orthologous group of chemosensory genes.

Additional file 3: A figure showing the correlation between protein distance and dN/dS ratio in each of the $O R, G R, I R$, and $O B P$ families. Figure $\mathbf{S} 1$. The two measurements of evolutionary rate are positively correlated in all chemosensory gene families. Scatter plots of protein distance and $\mathrm{dN} / \mathrm{dS}$ ratio for orthologous gene pairs in each of the $O R(A), G R(B), I R(C)$, and $O B P(D)$ families. Spearman's correlation (rho) between protein distances and $\mathrm{dN} / \mathrm{dS}$ ratios are shown for each family.

Additional file 4: A figure showing the distribution of radical and conservative amino acid substitutions on the predicted OR topology. Figure S2. Distribution of radical and conservative amino acid changes on predicted topological regions of OR genes. (A) Color coded representation of radical amino acid changes for each predicted topological regions of ORs. (B) Combined amino acid change per site for predicted transmembrane, intracellular, and extracellular regions. All values are averaged over all $O R$ genes.

Additional file 5: A table listing the number of radical and conservative amino acid substitutions in each of the predicted topological regions of each orthologous group of $O R$ genes.

Additional file 6: Reports the transcript abundances (in RPKMs) for every annotated AGAP in both species along with the "reliable log2" fold change (GFOLD value) for differences in abundance between species. 


\begin{abstract}
Additional file 7: A table listing the statistical comparisons of median and variance values of transcript enrichment between genes in different quartiles of $\mathrm{dN} / \mathrm{dS}$ ratios.
\end{abstract}

\section{Additional file 8: Reports the calculated odorant receptivity data to} select AgOrs.

Additional file 9: A figure showing the high reproducibility of RNA-seq results between biologically replicated antennal samples.

Figure S3. Correlation of RNAseq data between biologically replicated antennal samples. Scatter plot of the numbers of reads uniquely mapping to more than 13,000 individual An. gambiae genes (AGAPs) in each of two RNAseq samples. For each sample, antennal tissue was resected from the same cohort of non-blood fed An. gambiae females and was taken one day apart at identical, light-dark time points (ZT6). Approximately 800 individual antennae comprise each sample. Spearman's correlation (rho) between the two samples is shown.

\section{Competing interests}

The authors declare that they have no competing interests.

\section{Authors' contributions}

$D C R, X Z, R J P, A R$ and $L I Z$ discussed the experimental design and results. DCR and RJP preformed mosquito rearing, tissue collections and total RNA extractions. DCR and XZ preformed all bioinformatics analyses upon the RNAseq data sets. The AGC consortium generated the An. quadriannulatus reference genome assembly and authorized its use in this analysis in advance of the genome publication. DCR and XZ drafted the manuscript with subsequent contributions and revisions from DCR, XZ, RJP, AR and LJZ. All authors read and approved the final manuscript.

\section{Acknowledgments}

We thank Travis Clark, Chelsea Baker and the Vanderbilt Technologies for Advanced Genomics core facility for RNA sample preparation and Illumina sequencing. We are also indebted to the members of the Rokas/Zwiebel labs for helpful discussions and critical readings. This work was conducted in part using the resources of the Advanced Computing Center for Research and Education at Vanderbilt University and was supported by funds provided by the National Science Foundation (DEB-0844968) to AR, as well as grants from the Innovation and Discovery in Engineering And Science (IDEAS) program of Vanderbilt University and from the National Institutes of Health (NIAID, AI056402) to LJZ. DCR was supported by the NIDCD through an NRSA [F31 DC012991].

\section{The AGC Consortium}

Daniel E. Neafsey, Aaron M. Berlin, George K. Christophides, Frank H. Collins, Scott J. Emrich, Michael C. Fontaine, William Gelbart, Matthew W. Hahn, Paul I. Howell, Fotis C. Kafatos, Daniel Lawson, Marc A. T. Muskavitch, Sarah Young, Robert Waterhouse and Nora J. Besansky.

\section{Author details}

${ }^{1}$ Center for Human Genetics Research, Vanderbilt University Medical Center, Nashville, Tennessee, USA. ${ }^{2}$ Department of Biological Sciences, Vanderbilt University, Nashville, Tennessee, USA. ${ }^{3}$ Department of Pharmacology, Vanderbilt Brain Institute, Program in Developmental Biology and Institutes of Chemical Biology and Global Health, Vanderbilt University Medical Center, Nashville, Tennessee, USA.

Received: 11 July 2013 Accepted: 22 October 2013

Published: 1 November 2013

\section{References}

1. Coetzee M, Hunt RH, Wilkerson R: Anopheles coluzzii and Anopheles amharicus, new members of the Anopheles gambiae complex. Zootaxa 2013, 3619:246-274.

2. Scott TW, Takken W: Feeding strategies of anthropophilic mosquitoes result in increased risk of pathogen transmission. Trends Parasitol 2012, 28:114-121.

3. Takken W, Eling W, Hooghof J, Dekker T, Hunt R, Coetzee M: Susceptibility of Anopheles quadriannulatus Theobald (Diptera: Culicidae) to Plasmodium falciparum. T Roy Soc Trop Med H 1999, 93:578-580.
4. Habtewold T, Povelones M, Blagborough AM, Christophides GK: Transmission Blocking Immunity in the Malaria Non-Vector Mosquito Anopheles quadriannulatus Species A. PLoS Pathog 2008, 4:e1000070.

5. Kamali M, Xia A, Tu Z, Sharakhov IV: A new chromosomal phylogeny supports the repeated origin of vectorial capacity in malaria mosquitoes of the Anopheles gambiae complex. PLoS Pathog 2012, 8:e1002960.

6. White G: Comparative studies on sibling species of the Anopheles gambiae Giles complex (Dipt., Culicidae). III. The distribution, ecology, behaviour and vectorial importance of species D in Bwamba County, Uganda, with an analysis of biological, ecological, morphological and cytogenetical relationships of Ugandan species D*. Bull Entomol Res 1973, 63:65-97.

7. Dekker T, Takken W: Differential responses of mosquito sibling species Anopheles arabiensis and An-quadriannulatus to carbon dioxide, a man or a calf. Med Vet Entomol 1998, 12:136-140.

8. Pates H, Takken W, Curtis C, Huisman P, Akinpelu O, Gill G: Unexpected anthropophagic behaviour in Anopheles quadriannulatus. Med Vet Entomol 2001, 15:293-298.

9. Ayala F, Coluzzi M: Chromosome speciation: Humans, Drosophila, and mosquitoes. Proc Natl Acad Sci U S A 2005, 102:6535-6542.

10. Coluzzi M: A polytene chromosome analysis of the Anopheles gambiae species complex. Science 2002, 298:1415-1418.

11. Takken W, Knols BG: Odor-mediated behavior of Afrotropical malaria mosquitoes. Annu Rev Entomol 1999, 44:131-157.

12. Takken W, Verhulst NO: Host preferences of blood-feeding mosquitoes. Annu Rev Entomol 2013, 58:433-453.

13. Pitts RJ, Fox AN, Zwiebel L: A highly conserved candidate chemoreceptor expressed in both olfactory and gustatory tissues in the malaria vector Anopheles gambiae. Proc Natl Acad Sci U S A 2004, 101:5058-5063.

14. Melo ACA, Rützler M, Pitts RJ, Zwiebel LJ: Identification of a chemosensory receptor from the yellow fever mosquito, Aedes aegypti, that is highly conserved and expressed in olfactory and gustatory organs. Chem Senses 2004, 29:403-410.

15. Kwon H-W, Lu T, Rützler M, Zwiebel LJ: Olfactory responses in a gustatory organ of the malaria vector mosquito Anopheles gambiae. Proc Natl Acad Sci USA 2006, 103:13526-13531.

16. Schymura D, Forstner M, Schultze A, Kröber T, Swevers L, latrou K, Krieger J: Antennal expression pattern of two olfactory receptors and an odorant binding protein implicated in host odor detection by the malaria vector Anopheles gambiae. Int J Biol Sci 2010, 6:614-626.

17. Jones WD, Nguyen T-AT, Kloss B, Lee KJ, Vosshall LB: Functional conservation of an insect odorant receptor gene across 250 million years of evolution. Curr Biol 2005, 15:R119-R121.

18. Xia Y, Zwiebel LJ: Identification and characterization of an odorant receptor from the West Nile virus mosquito, Culex quinquefasciatus. Insect Biochem Mol Biol 2006, 36:169-176.

19. Qiu YT, van Loon JJA, Takken W, Meijerink J, Smid HM: Olfactory Coding in Antennal Neurons of the Malaria Mosquito, Anopheles gambiae. Chem Senses 2006, 31:845-863.

20. Takken W, van Loon J, Adam W: Inhibition of host-seeking response and olfactory responsiveness in Anopheles gambiae following blood feeding. J Insect Physiol 2001, 47:303-310.

21. Fox AN, Pitts RJ, Robertson HM, Carlson JR, Zwiebel LJ: Candidate odorant receptors from the malaria vector mosquito Anopheles gambiae and evidence of down-regulation in response to blood feeding. Proc Natl Acad Sci USA 2001, 98:14693-14697.

22. Hill CA, Fox AN, Pitts RJ, Kent LB, Tan PL, Chrystal MA, Cravchik A, Collins FH, Robertson HM, Zwiebel LJ: G Protein-Coupled receptors in Anopheles gambiae. Science 2002, 298:176-178.

23. Lu Q, Lu T, Qiu YT, Wang G, Kwon JY, Rutzler M, Kwon HW, Pitts RJ, van Loon JJA, Takken W, Carlson JR, Zwiebel $\sqcup$ : Odor coding in the Maxillary Palp of the malaria vector mosquito Anopheles gambiae. Curr Biol 2007, 17:1533-1544.

24. Xia Y, Wang G, Buscariollo D, Pitts RJ, Wenger H, Zwiebel L: The molecular and cellular basis of olfactory-driven behavior in Anopheles gambiae larvae. Proc Natl Acad Sci USA 2008, 105:6433-6438.

25. Carey AF, Wang G, Su C-Y, Zwiebel LJ, Carlson JR: Odorant reception in the malaria mosquito Anopheles gambiae. Nature 2010:1-38.

26. Wang G, Carey AF, Carlson JR, Zwiebel LJ: Molecular basis of odor coding in the malaria vector mosquito Anopheles gambiae. Proc Natl Acad Sci USA 2010, 107:4418-4423. 
27. Pitts RJ, Rinker DC, Jones PL, Rokas A, Zwiebel LJ: Transcriptome profiling of chemosensory appendages in the malaria vector Anopheles gambiae reveals tissue- and sex-specific signatures of odor coding. BMC Genomics 2011, 12:271.

28. Rinker DC, Pitts RJ, Zhou X, Suh E, Rokas A, Zwiebel LJ: Blood meal-induced changes to antennal transcriptome profiles reveal shifts in odor sensitivities in Anopheles gambiae. Proc Natl Acad Sci USA 2013, 110:1-9.

29. Hittinger $C T$, Johnston M, Tossberg JT, Rokas A: Leveraging skewed transcript abundance by RNA-Seq to increase the genomic depth of the tree of life. Proc Natl Acad Sci USA 2010, 107:1476-1481.

30. Kopp A, Barmina O, Hamilton AM, Higgins L, McIntyre LM, Jones CD: Evolution of gene expression in the Drosophila olfactory system. Mol Biol Evol 2008, 25:1081-1092.

31. Xu PX, Zwiebel LJ, Smith DP: Identification of a distinct family of genes encoding atypical odorant-binding proteins in the malaria vector mosquito, Anopheles gambiae. Insect Mol Biol 2003, 12:549-560.

32. Liu C, Pitts RJ, Bohbot JD, Jones PL, Wang G, Zwiebel LJ: Distinct Olfactory Signaling Mechanisms in the Malaria Vector Mosquito Anopheles gambiae. PLOS Biol 2010, 8:e1000467.

33. Croset V, Rytz R, Cummins S, Budd A, Brawand D, Kaessmann H, Gibson TJ, Benton R: Ancient Protostome Origin of Chemosensory lonotropic Glutamate Receptors and the Evolution of Insect Taste and Olfaction. PLoS Genet 2010, 6:e1001064.

34. Rinker DC, Jones PL, Pitts RJ, Rützler M, Camp G, Sun L, Xu P, Dorset DC, Weaver D, Zwiebel LJ: Novel high-throughput screens of Anopheles gambiae odorant receptors reveal candidate behaviour-modifying chemicals for mosquitoes. Physiol Entomol 2012, 37:33-41.

35. Hallem EA, Fox AN, Zwiebel LJ, Carlson JR: Olfaction: mosquito receptor for human-sweat odorant. Nature 2004, 427:212-213.

36. Meijerink J, Braks M, Brack AA, Adam W, Dekker T, Posthumus MA, Beek TAV, Van Loon JJA: Identification of Olfactory Stimulants for Anopheles gambiae from Human Sweat Samples. J Chem Ecol 2000, 26:1367-1382.

37. Meijerink J, Braks MA, Van Loon JJ: Olfactory receptors on the antennae of the malaria mosquito Anopheles gambiae are sensitive to ammonia and other sweat-borne components. J Insect Physiol 2001, 47:455-464.

38. Braks MA, Takken W: Incubated Human Sweat but not Fresh Sweat Attracts the Malaria Mosquito Anopheles gambiae sensu stricto. J Chem Ecol 1999, 25:663-672.

39. Thorne N, Chromey C, Bray S, Amrein H: Taste perception and coding in Drosophila. Curr Biol 2004, 14:1065-1079.

40. Dekker T, Geier M, Cardé RT: Carbon dioxide instantly sensitizes female yellow fever mosquitoes to human skin odours. J Exp Bio/ 2005, 208:2963-2972.

41. Ai M, Min S, Grosjean Y, Leblanc C, Bell R, Benton R, Suh GSB: Acid sensing by the Drosophila olfactory system. Nature 2010, 468:691-695.

42. Biessmann $H$, Nguyen QK, Le D, Walter MF: Microarray-based survey of a subset of putative olfactory genes in the mosquito Anopheles gambiae. Insect Mol Biol 2005, 14:575-589.

43. Pelletier J, Guidolin A, Syed Z, Cornel AJ, Leal WS: Knockdown of a Mosquito Odorant-binding Protein Involved in the Sensitive Detection of Oviposition Attractants. J Chem Ecol 2010, 36:245-248.

44. Biessmann $H$, Andronopoulou E, Biessmann MR, Douris V, Dimitratos SD, Eliopoulos E, Guerin PM, latrou K, Justice RW, Kröber T, Marinotti O, Tsitoura P, Woods DF, Walter MF: The Anopheles gambiae odorant binding protein 1 (AgamOBP1) mediates indole recognition in the antennae of female mosquitoes. PLOS ONE 2010, 5:e9471.

45. Zhou X, Slone JD, Rokas A, Berger SL, Liebig J, Ray A, Reinberg D, Zwiebel LJ: Phylogenetic and Transcriptomic Analysis of Chemosensory Receptors in a Pair of Divergent Ant Species Reveals Sex-Specific Signatures of Odor Coding. PLoS Genet 2012, 8:e1002930.

46. Robertson HM, Warr CG, Carlson JR: Molecular evolution of the insect chemoreceptor gene superfamily in Drosophila melanogaster. Proc Natl Acad Sci USA 2003, 100(Suppl 2):14537-14542.

47. Sánchez-Gracia A, Vieira FG, Almeida FC, Rozas J: Comparative Genomics of the Major Chemosensory Gene Families in Arthropods. John Wiley \& Sons, Ltd: Chichester, UK; 2001.

48. Gilad Y, Wiebe V, Przeworski M, Lancet D, Pääbo S: Loss of Olfactory receptor genes coincides with the acquisition of full trichromatic vision in primates. PLOS Biol 2004, 2:e5.
49. Stensmyr M, Dekker T, Hansson B: Evolution of the olfactory code in the Drosophila melanogaster subgroup. P Roy Soc Lond B Bio 2003, 270:2333-2340.

50. Dekker T, Ibba I, Siju KP, Stensmyr MC, Hansson BS: Olfactory shifts parallel superspecialism for toxic fruit in Drosophila melanogaster siblingD. sechellia. Curr Biol 2006, 16:101-109.

51. Mcbride CS, Arguello JR, O'meara BC: Five Drosophila genomes revea nonneutral evolution and the signature of host specialization in the chemoreceptor superfamily. Genetics 2007, 177:1395-1416.

52. McBride CS: Rapid evolution of smell and taste receptor genes during host specialization in Drosophila sechellia. Proc Natl Acad Sci USA 2007, 104:4996-5001

53. Torre A d, Costantini C, Besansky NJ, Caccone A, Petrarca V, Powell JR, Coluzzi M: Speciation within Anopheles gambiae-the glass is half full. Science 2002, 298:115-117.

54. Obbard DJ, Maclennan J, Kim K-W, Rambaut A, O'Grady PM, Jiggins FM: Estimating divergence dates and substitution rates in the Drosophila phylogeny. Mol Biol Evol 2012, 29:3459-3473.

55. Bernays EA, Chapman RE: Patterns of Host-Plant Use. In Host-Plant Selection by Phytophagous Insects. New York: Chapmand and Hall; 1994:4-13.

56. Smith NGC: Are radical and conservative substitution rates useful statistics in molecular evolution? J Mol Evol 2003, 57:467-478.

57. Hanada K, Shiu SH, LI WH: The nonsynonymous/synonymous substitution rate ratio versus the radical/conservative replacement rate ratio in the evolution of mammalian genes. Mol Biol Evol 2007, 24:2235-2241.

58. Nichols AS, Luetje CW: Transmembrane segment 3 of drosophila melanogaster odorant receptor subunit $85 \mathrm{~B}$ contributes to ligand-receptor interactions. J Bio Chem 2010, 285:11854-11862.

59. Nakagawa T, Pellegrino M, Sato K, Vosshall LB, Touhara K: Amino acid residues contributing to function of the heteromeric insect olfactory receptor complex. PLOS ONE 2012, 7:e32372.

60. DeGennaro M, McBride CS, Seeholzer L, Nakagawa T, Dennis EJ, Goldman C, Jasinskiene N, James AA, Vosshall LB: orco mutant mosquitoes lose strong preference for humans and are not repelled by volatile DEET. Nature 2013, 498:487-491.

61. Larsson MC, Domingos Al, Jones WD, Chiappe ME, Amrein $H_{\text {, }}$ Vosshall LB: Or83b encodes a broadly expressed odorant receptor essential for Drosophila olfaction. Neuron 2004, 43:703-714.

62. Pitts RJ, Zwiebel $\amalg$ : Antennal sensilla of two female anopheline sibling species with differing host ranges. Malar J 2006, 5:26.

63. Cork A, Park KC: Identification of electrophysiologically-active compounds for the malaria mosquito, Anopheles gambiae, in human sweat extracts. Med Vet Entomol 1996, 10:269-276.

64. Bohbot JD, Jones PL, Wang G, Pitts RJ, Pask GM, Zwiebel LJ: Conservation of indole responsive odorant receptors in mosquitoes reveals an ancient olfactory trait. Chem Senses 2010, 36:149-160.

65. Bernier UR, Kline DL, Barnard DR, Schreck CE, Yost RA: Analysis of human skin emanations by gas chromatography/Mass spectrometry. 2. identification of volatile compounds that are candidate attractants for the yellow fever mosquito (Aedesaegypti). Anal Chem 2000, 72:747-756.

66. Hughes DT, Pelletier J, Luetje CW, Leal WS: Odorant receptor from the Southern House mosquito narrowly tuned to the Oviposition attractant Skatole. J Chem Ecol 2010, 36:797-800.

67. Pelletier J, Hughes DT, Luetje CW, Leal WS: An odorant receptor from the southern house mosquito Culex pipiens quinquefasciatus sensitive to oviposition attractants. PLOS ONE 2010, 5:e10090.

68. Millar JG, Chaney JD, Mulla MS: Identification of oviposition attractants for Culex quinquefasciatus from fermented Bermuda grass infusions. J Am Mosquito Contr 1992, 8:11-17.

69. Kostelc JG, Preti G, Zelson PR, Stoller NH, Tonzetich J: Salivary volatiles as indicators of periodontitis. J Periodont Res 1980, 15:185-192.

70. Pates H, Takken W, Stuke K, Curtis C: Differential behaviour of Anopheles gambiae sensu stricto (Diptera Culicidae) to human and cow odours in the laboratory. Bull Entomol Res 2001, 91:289-296.

71. Altschul SF, Madden TL, Schäffer AA, Zhang J, Zhang Z, Miller W, Lipman DJ: Gapped BLAST and PSI-BLAST: a new generation of protein database search programs. Nucleic Acids Res 1997, 25:3389-3402.

72. Birney E: GeneWise and Genomewise. Genome Res 2004, 14:988-995. 
73. Katoh K, Standley DM: MAFFT multiple sequence alignment software Version 7: Improvements in performance and usability. Mol Biol Evol 2013, 30:772-780

74. Capella-Gutierrez S, Silla-Martinez JM, Gabaldon T: trimAl: a tool for automated alignment trimming in large-scale phylogenetic analyses. Bioinformatics 2009, 25:1972-1973.

75. Stamatakis A: RAxML-VI-HPC: maximum likelihood-based phylogenetic analyses with thousands of taxa and mixed models. Bioinformatics 2006, 22:2688-2690.

76. Yang Z: PAML 4: Phylogenetic analysis by maximum likelihood. Mol Biol Evol 2007, 24:1586-1591.

77. Zhang J: Rates of conservative and radical nonsynonymous nucleotide substitutions in mammalian nuclear genes. J Mol Evol 2000, 50:56-68.

78. Bernsel $A$, Viklund $H$, Hennerdal A, Elofsson A: TOPCONS: consensus prediction of membrane protein topology. Nucleic Acids Res 2009, 37(Web Serve):W465-W468.

79. Grabherr MG, Haas BJ, Yassour M, Levin JZ, Thompson DA, Amit I, Adiconis X, Fan L, Raychowdhury R, Zeng Q, Chen Z, Mauceli E, Hacohen N, Gnirke A, Rhind N, di Palma F, Birren BW, Nusbaum C, Lindblad-Toh K, Friedman N, Regev A: Full-length transcriptome assembly from RNA-Seq data without a reference genome. Nature Biotech 2011, 29:644-652.

80. Li L, Stoeckert CJ, Roos DS Jr: OrthoMCL: identification of ortholog groups for eukaryotic genomes. Genome Res 2003, 13:2178-2189.

81. Trapnell C, Roberts A, Goff L, Pertea G, Kim D, Kelley DR, Pimentel H, Salzberg SL, Rinn JL, Pachter L: Differential gene and transcript expression analysis of RNA-seq experiments with TopHat and Cufflinks. Nature Publishing Group 2012, 7:562-578.

82. Feng J, Meyer CA, Wang Q, Liu JS, Liu SX, Zhang Y: GFOLD: a generalized fold change for ranking differentially expressed genes from RNA-seq data. Bioinformatics 2012, 28:2782-2788

83. Mortazavi A, Williams BA, Mccue K, Schaeffer L, Wold B: Mapping and quantifying mammalian transcriptomes by RNA-Seq. Nat Meth 2008 , 5:621-628.

doi:10.1186/1471-2164-14-749

Cite this article as: Rinker et al: Antennal transcriptome profiles of anopheline mosquitoes reveal human host olfactory specialization in Anopheles gambiae. BMC Genomics 2013 14:749.

\section{Submit your next manuscript to BioMed Central and take full advantage of:}

- Convenient online submission

- Thorough peer review

- No space constraints or color figure charges

- Immediate publication on acceptance

- Inclusion in PubMed, CAS, Scopus and Google Scholar

- Research which is freely available for redistribution 